\title{
Dynamic Nuclear Polarization using short-lived photo-excited triplet states: experiments and applications
}

\section{P. HAUTLE*, N. NIKETIC, B. VAN DEN BRANDT, J. KOHLBRECHER, W. TH. WENCKEBACH}

Paul Scherrer Institut, CH-5232 Villigen PSI

E-mail: patrick.hautledpsi.ch

\begin{abstract}
In DNP experiments the high polarization of an electronic spin system is transferred by suitable microwave irradiation to the nuclear spin system. In usual classical schemes ground state paramagnetic centers are introduced into the sample as dopants and their electron spin polarization is determined thermally. This requires temperatures of $1 \mathrm{~K}$ or lower and a magnetic field of several Tesla. As an alternative, short-lived photo-excited triplet states are highly polarized independent of temperature and field. But classical DNP, driven by weak cw microwave irradiation is too slow and one has to resort to pulsed DNP using strong microwave fields. During the last years we have pushed the development of "triplet DNP" in view of applications in neutron scattering. By optimizing several crucial parameters, proton polarization values of above $70 \%$ are now routinely achieved in naphthalene single crystals doped with pentacene in a field of $0.36 \mathrm{~T}$ at about $40 \mathrm{~K}$. We have demonstrated that triplet DNP is well suited to build a neutron spin filter to polarize a neutron beam in a wide energy range and to perform polarization analysis in small angle neutron scattering in a magnetic field.
\end{abstract}

XVIth International Workshop in Polarized Sources, Targets, and Polarimetry

14-18 September 2015

Ruhr-University Bochum, Germany

\footnotetext{
* Speaker.
} 


\section{Introduction}

As an alternative to thermally polarized ground state paramagnetic centers, short lived photoexcited triplet states can also provide the polarized electron spins for the DNP process [四]. A proven system for triplet DNP is a naphthalene molecular host crystal doped with a small concentration of pentacene guest molecules, typically a few $10^{-5} \mathrm{~mol} / \mathrm{mol}$ (Fig. W). When illuminating pentacene with a short optical pulse the molecule is excited from a singlet ground state $S_{0}$ to the lowest excited singlet state $S_{1}$. From $S_{1}$ the system may decay back to ground state under the emission of fluorescence, or decays via intersystem crossing (ISC) to the lowest triplet state $T_{1}$ with a strongly aligned electron spin $S=1$. While the life time of the lowest excited singlet state $S_{1}$ is only about $20 \mathrm{~ns}$, the life time of the lowest triplet state $T_{1}$ is of the order $50 \mu \mathrm{s}$ [ [2], allowing its use for DNP. Furthermore, the electron spin lattice relaxation time (typically some $100 \mu \mathrm{s}$ ) is much longer than the lifetime of the triplet state. Hence, the triplet electron spin polarization is not only independent of temperature and strength of the applied field, but also almost completely maintained during the life time of the triplet state. It depends only on the direction of the external field; single crystals are indispensable to achieve high nuclear polarizations. In an external magnetic field two electron spin resonance (ESR) lines are observed at $\sim 0.3 \mathrm{~T}$ corresponding to the transitions $S_{z}=-1 \leftrightarrow 0$ and $S_{z}=0 \leftrightarrow+1$. For $B \| X$ the ESR lines are separated by about $1.5 \mathrm{GHz}$. Also the $S_{z}=0$ level is strongly favored. In triplet DNP only one of the two ESR transitions is used and the two energy levels involved may be regarded as an effective spin- $\frac{1}{2}$ system with an "effective" electron spin polarization $P \sim 0.9$ [[]].

The short life time of the triplet state requires a pulsed microwave irradiation scheme to resonantly transfer this high electron polarization to the nuclear spins. This is most effectively done by means of the integrated solid effect (ISE) [ [U, [], 团]. In this method an externally applied magnetic field is adiabatically swept over the in-homogeneously broadened ESR line (typically in $10 \mu \mathrm{s}$ ) under microwave irradiation, which allows all electron spin to constructively participate in the polarization transfer. As in classical DNP, the nuclear polarization thus created is localized around the triplet guest molecules and then spreads out further over the host molecules via spin diffusion. Provided the proton spin-lattice relaxation is long enough, the cycle can be repeated many times and the proton spin polarization is accumulated and can approach the electron spin polarization. Since the proton spin-lattice relaxation time in naphthalene is already very long at liquid nitrogen temperature ( $>24 \mathrm{~h}$ in a field of $0.3 \mathrm{~T}$ ), triplet DNP experiments need moderate cryogenic means only.

The pioneering work on this system has been performed by the group of Wenckebach in the late eighties in Leiden [四]. Only much later Japanese researchers at Kyoto University took up the subject again in view of a polarised target application [可. Work was continued by NMR specialists at Kyoto and Osaka University who further explored the method to apply it in the field of NMR spectroscopy [ $[0,0]$ and quantum information science [ [8]. The method has recently also taken its first step from a pure laboratory setup to a working polarised target system for the use on radioactive ion beams $[Q,[0]$. With the important progress we have achieved during the last few years at PSI, the technique has reached an appreciable level and is certainly mature to be transferred into applications, e.g. a neutron spin filter device in our case. 

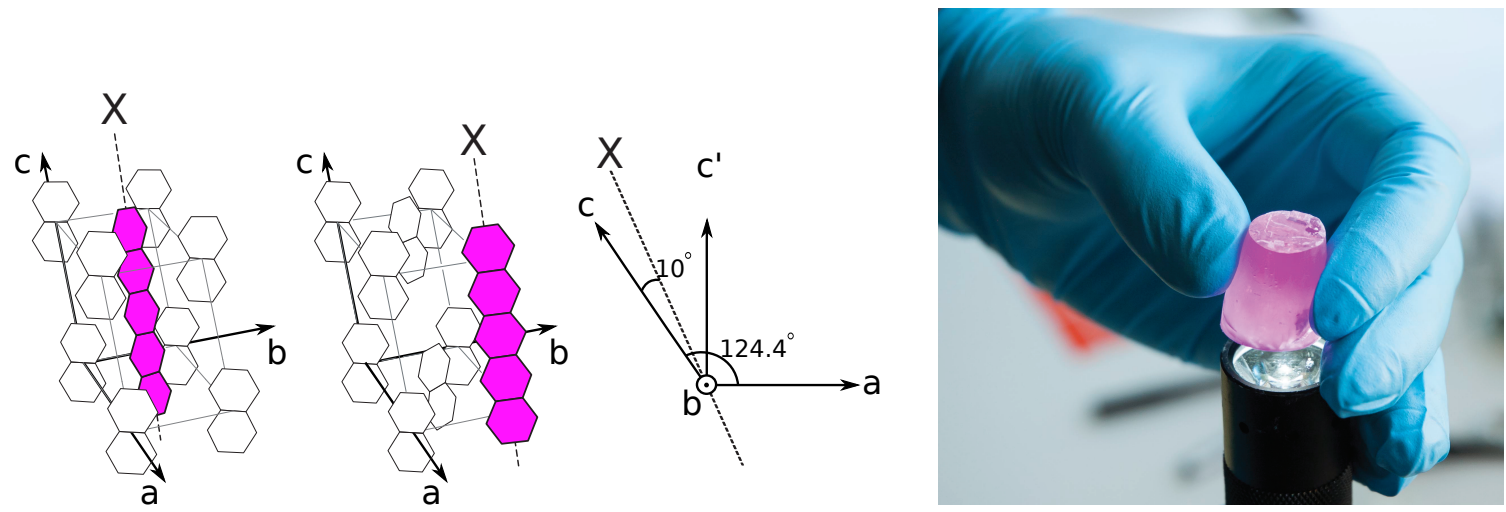

Figure 1: Left: The crystal structure of pure naphthalene is monoclinic with a unit cell with axes $a \sim 8.1 \AA$, $b \sim 5.9 \AA, c \sim 8.6 \AA$ and $\beta=124.4^{\circ}$ [ए]]. The $a$ - $b$ plane is the cleavage plane. Two naphthalene molecules can be replaced at two positions by one pentacene molecule whose $X$-axis lies in the $a$ - $c$ plane at an angle of $10^{\circ}$ to the $c$-axis. Right: Large single crystals of naphthalene doped with pentacene can be grown with a self seeding Bridgeman method

\section{Apparatus}

Two X-band $(9.4 \mathrm{GHz})$ triplet-DNP systems have been set up. They consist of a pulsed electron spin resonance spectrometer, which has specifications adequate to study triplet-state properties of systems like pentacene-doped naphthalene and also allows performing different pulse DNP schemes. The nuclear polarization is monitored with a pulse NMR system. The design of the dielectric ring microwave cavity allows performing an in situ nuclear polarization measurement with an integrated NMR coil. One triplet DNP system is dedicated to the spin filter application and has been setup in a compact Varian electro magnet system capable to go up to $0.65 \mathrm{~T}$. The system is suitable to polarize sample sizes of about $5 \times 5 \times 5 \mathrm{~mm}^{3}$. A home-made ${ }^{4} \mathrm{He}$ flow cryostat with optical access and suited for ESR/NMR was designed, that operates at temperatures between $4 \mathrm{~K}$ and $300 \mathrm{~K}$. It is especially suited for experiments in neutron beams, i.e. the part where the neutrons pass, is completely made of aluminium, leading to a transmission of above $90 \%$. For the optical excitation two different laser systems are used. The light of both systems can be simultaneously coupled with high efficiency into a high numerical aperture multi-mode fiber that transports the light over long distances (up to $20 \mathrm{~m}$ ), allowing to conveniently separate the laser system from the rest of the apparatus. The fiber is attached at the other end to an optical stage at the bottom of the cryostat, which collimates the unpolarized light onto the sample crystal. The first laser source is well suited for studies of the light excitation. It consists of a frequency tripled Nd:YAG (SpectraPhysics Pro270) followed by an optical parametric oscillator (GWU premiScan). The OPO allows tuning of the irradiation wavelength in a range from $400 \mathrm{~nm}$ to above $600 \mathrm{~nm}$. The second source is an infrared laser diode-pumped Yb:YAG disk laser (Jenlas disk IR50) designed for industrial purpose but well suited for a fast polarization enhancement. Its output is frequency-doubled to $515 \mathrm{~nm}$ with conversion efficiency of 0.3 in a lithium triborate (LBO) nonlinear crystal at room temperature using critical phase-matching. The technical details of the triplet system and its performance are described in [12].

The samples used for DNP in this apparatus are typically cubes of about 4 to $5 \mathrm{~mm}$ side length. 
They are cut out of several cubic centimetre large high quality naphthalene single crystals doped with pentacene (see Fig. W). Naphthalene is extensively zone refined and the pentacene is purified by sublimation. The crystals are grown with a home built apparatus, employing a self-seeding vertical Bridgman technique [[13]. They typically contain pentacene with a concentration between 2 and $4 \times 10^{-5} \mathrm{~mol} / \mathrm{mol}$, determined by optical transmission spectroscopy. Keeping the concentration levels in this range still assures a high crystal quality, which has been confirmed by neutron diffraction and furthermore proves to be well adapted to the available laser pulse energies. The samples are normally cut such that their bottom/top is in the crystals $a-c$ plane with one side parallel to the pentacene molecular $X$-axis. With the crystal $b$-axis vertical, the sample is then clamped to a Kel-F (chlorotrifluoroethylene) rod that is attached to the sample stick. The latter can be rotated in order to align the pentacene $X$-axis, where all molecules are magnetically equivalent, with the horizontal magnetic holding field. A fine alignment can be done by rotating the magnet. The misalignment due to improper cutting and mounting is estimated to be around $2^{\circ}$. The light is irradiating the sample from below along the crystal $b$-axis.

\section{Recent progress in triplet DNP}

The breakthrough of above $70 \%$ proton polarization recently achieved [44] is the consequence of the experimental and theoretical work done in the past two years. We introduced the use of fully deuterated pentacene- $d_{14}$ as dopant and demonstrated that the polarization efficiency can be boosted in combination with the highly efficient laser source [15]. The narrower ESR line and the prolonged triplet state lifetime, as compared to initially used protonated pentacene dopants, results in a considerably higher average triplet electron polarization that is transferred in each cycle of the integrated solid effect (ISE) sequence. Running the sequence at $4 \mathrm{kHz}$ a proton polarization build-up of more that $1 \% / \mathrm{min}$ can be achieved [प2]. On the basis of this initial work, Japanese researchers recently confirmed an increase in efficiency by using deuterated pentacene to polarize protons in a perdeuterated p-terphenyl host [ए], ㅁ] ]. The integrated solid effect (ISE), originally introduced by Henstra et al. [四], is recognized as the most adequate polarization transfer scheme for the short-lived paramagnetic triplet states of pentacene in naphthalene crystals. However, a complete theoretical understanding, that could guide the optimum setting of the experimental parameters, was so far lacking. Contrary to classical DNP driven by weak cw microwave irradiation [ए8], in ISE one uses strong pulsed microwave fields and the polarization transfer is a coherent process, which requires a completely different theoretical approach. Henstra et al. have developed the complete theoretical description of ISE that is able to calculate the polarization transfer [B]]. The theory was validated in an extensive series of experiments [团]. The optimum parameter settings for the ISE, i.e. the optimum combination of sweep speed and microwave power, can now be established for a specific experimental situation. This resulted in a record proton polarization of above 0.7 that has been achieved in several experiments with different pentacene- $d_{14}$ doped naphthalene single crystals $\left(\sim 5 \times 5 \times 5 \mathrm{~mm}^{3}\right.$ size $)$ in a field of $0.36 \mathrm{~T}$, using the simple flow cryostat for cooling (see Fig. 凹, left). The polarization values were monitored by NMR and calibrated by neutron transmission, as described in [ए]]. The latter method was further used to confirm that the polarization in the sample is homogeneous along the laser beam path. 

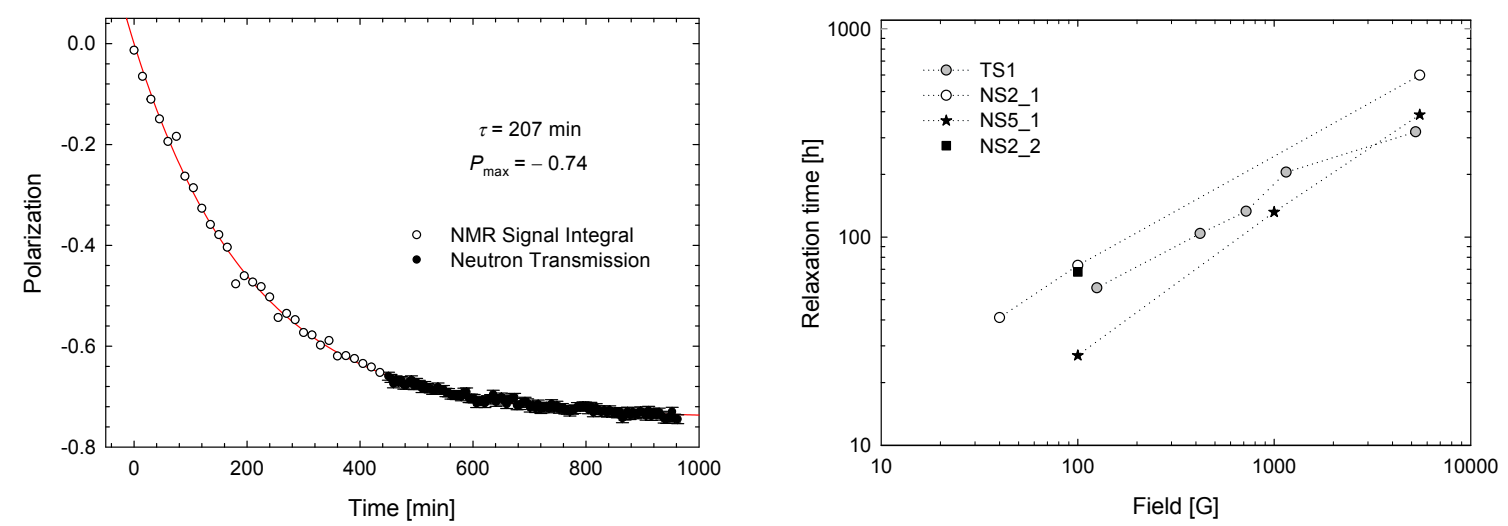

Figure 2: Left: Typical polarization build-up at an ISE repetiton rate of $1 \mathrm{kHz}$. Right: Relaxation times for various crystals at a temperature of $T=25 \mathrm{~K}$ as function of the magnetic holding field. The best crystal exhibits relaxation times of $41 \mathrm{~h}$ at $40 \mathrm{G}, 73 \mathrm{~h}$ at $100 \mathrm{G}$, and above $600 \mathrm{~h}$ at $5500 \mathrm{G}$.

Furthermore, the apparatuses and procedures to grow large high quality pentacene doped naphthalene single crystals have been constantly improved. Extensive zone refinement cleaning of the naphthalene base material over several month time, as well as performing the crucial preparation steps in a glove box under nitrogen atmosphere, are key to obtain samples that have long nuclear relaxation times even at very low field (see Fig. \2, right).

\section{Applications: Neutron spin filtering}

Our efforts developing the triplet DNP method are driven by the aim to implement it into a compact apparatus to spin filter neutrons. The working principle of a polarized proton spin filter is based on the fact that the singlet cross section for neutron-proton scattering is much higher than the triplet cross section [[Z] $]$. Hence, neutrons polarized anti-parallel to the protons are much stronger scattered than those polarized parallel. This applies for coherent and incoherent scattering. It is customary to write the cross section as

$$
\sigma_{ \pm}=\sigma_{0} \pm \sigma_{p} P
$$

where the + sign and the - sign stand for the two eigenstates of the neutron spin with respect to the direction of the proton spin polarization. The second term is the product of the "polarization cross-section' $\sigma_{p}$ and the proton spin polarization $P$. The first term $\sigma_{0}$ is independent of the neutron spin polarization and either independent of $P$ or a symmetric function of $P$.

The transmissions of the two spin components of the unpolarized neutron beam through a spin filter with a proton density $N$ and a thickness $d$ are given by

$$
T_{ \pm}=\frac{1}{2} \exp \left[-\left(\sigma_{0} \pm \sigma_{p} P N d\right)\right] .
$$

Thus an initially unpolarized neutron beam acquires a polarization

$$
A=\frac{T_{+}-T_{-}}{T_{+}+T_{-}}=\tanh \left(\sigma_{p} P N d\right),
$$


which is called the filter analyzing power. The thicker the filter, the higher the filter analyzing power for a given $\left(\sigma_{p} N\right)$, and the lower the transmission

$$
T=T_{+}+T_{-}=\exp \left(-\sigma_{0} N d\right) \cosh \left(\sigma_{p} P N d\right) .
$$

of the neutron beam. Note that for a given thickness $d$, the total transmission $T$ increases with the filter polarization $P$.

In a first proof of principle experiment we demonstrated that the triplet DNP method can be used to build a reliably working spin filter for neutrons operating at $0.3 \mathrm{~T}$ at a temperature of $100 \mathrm{~K}$ [ㅁ] $]$. Furthermore the wavelength dependent polarization cross section $\sigma_{p}(\lambda)$ for naphthalene has been determined over the spectrum of the white cold beam to high precision with a moderate proton polarization only. The knowledge of $\sigma_{p}(\lambda)$ then provides a method complementary to NMR, to characterize the naphthalene samples DNP performance, i.e. the filter polarization is readily determined with a high absolute precision (around $3 \%$ ) via a simple measurement of the transmission of the two neutron beam polarization states. Moreover, the neutron beam can be used to measure the proton spin polarization as a function of position in the naphthalene sample, which we use as a method to check the polarization homogeneity in the crystal.

Making use of a focus de-focus neutron guide arrangement, we have then shown that a broadband spin filter for a typical beam size can be realized using only a small filter crystal. We have integrated the highly polarized spin filter in a focus de-focus neutron guide system and could increase its effective cross section by a factor 35, corresponding to an area of $18 \times 18 \mathrm{~mm}^{2}$. Knowing the relevant polarization cross section the filters performance can be estimated using the equations given above. Taking the so called figure of merit defined by $Q=A^{2} \times T$ as a guideline, the triplet spin filter compares well against the most advanced ${ }^{3} \mathrm{He}$ spin filters [14]].

The insensitivity of the triplet DNP method to a magnetic environment opens the possibility of a tight integration of the filter with the sample environment in an analyzer arrangement. We demonstrated very recently that the triplet spin filter can be readily used for polarization analysis in small angle neutron scattering (SANS) on a nano-structured magnetic alloy. The few experiments of this type were performed with ${ }^{3} \mathrm{He}$ filters [2], 22, [23] that had to be placed in a homogeneous field cavity ("magic box") at long distance to the sample to prevent rapid relaxation of the ${ }^{3} \mathrm{He}$ polarization in a stray field of the sample magnet. As the triplet spin filter needs to be operated in a magnetic field we adopted the opposite strategy and integrated the sample with the spin filter analyzer. The sample, a small platelet of CuNiFe, was placed outside of the flow cryostat $32 \mathrm{~mm}$ before the sample, i.e. it was at room temperature but still in the homogeneous magnetic field of the electromagnet. The distance between sample and analyzer was sufficiently small to cover the necessary angular range of the scattering even with the relatively small analyzer cross section defined by a $3 \times 3 \mathrm{~mm}^{2}$ aperture placed in the front of the polarized naphthalene crystal. At the wavelength of $0.6 \mathrm{~nm}$ the accessible q-range was $0.41 \mathrm{~nm}^{-1}$. SANS measurements were performed at different fields for the four spin channels and already the raw images display the pronounced intensity distribution expected for magnetic scattering (Fig. (B)). This spin dependent scattering data could be understood using simulations based on a model of superparamagnetic particles, which are ordered in a simple cubic paracrystalline lattice. The excellent agreement of the experimental data with these simulations well demonstrates the usability of the triplet spin filter as analyzer. For more details we refer to our publication [24]. 


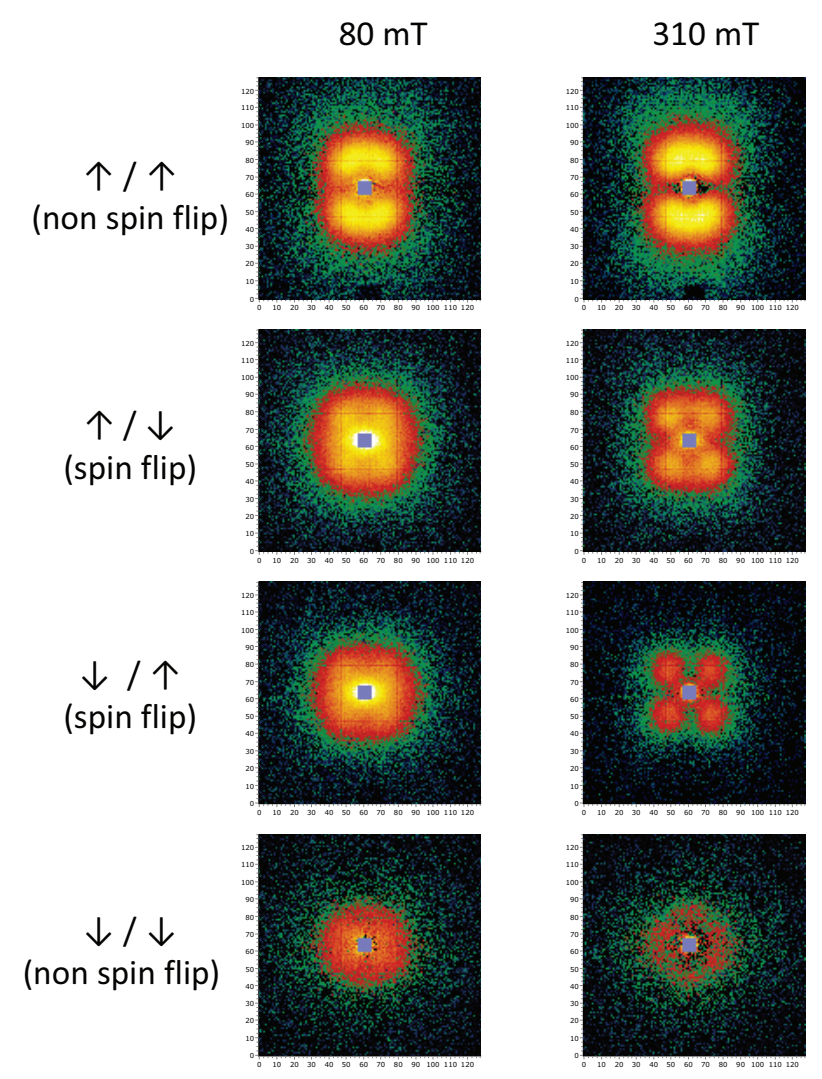

Figure 3: SANS images at $11 \mathrm{~m}$ detector distance of a CuNiFe sample taken at two different non-saturating magnetic fields for the 4 different combinations of the polarization states of incoming and scattered neutrons (indicated on the left with arrows). The images represent raw data and are not yet corrected for so called spin leakage that results from an analyzer efficiency smaller than unity

\section{Conclusion}

We have considerably pushed the development of the triplet DNP method and used it to build a novel type of neutron spin filter. The device has been operated very reliably in several 2 to 4 week long experiments on neutron beams. The high proton polarization achieved in naphthalene doped with pentacene- $\mathrm{d}_{14}$ using moderate cryogenics and a small magnet lets it become a serious alternative to the well-established methods of polarizing neutrons, super mirror polarizers and polarized gaseous ${ }^{3} \mathrm{He}$. Analogous to a ${ }^{3} \mathrm{He}$ filter, it can be used as a neutron polarizer or as a neutron polarization analyzer. Its tremendous bandwidth in neutron energy is of key importance for the former, whereas its insensitivity to a magnetic environment allows a tight integration with the sample environment in an analyzer arrangement.

To further improve the filter performance and expand its possibilities, we have started work on increasing the filter size. This requires a redesign of the ESR resonant structure that will be operated at a lower microwave frequency at around $5 \mathrm{GHz}$. 


\section{References}

[1] A. Henstra, T.-S. Lin, J. Schmidt, W. Th. Wenckebach, Chem. Phys. Lett. 165 (1990) 6-10.

[2] A.J. van Strien, J. Schmidt, Chem. Phys. Lett. 70 (1980) 513.

[3] A. Henstra, W. Th. Wenckebach, Mol. Phys. 112 (2014) 1761.

[4] T.R. Eichhorn, B. van den Brandt, P. Hautle, A. Henstra, W. Th. Wenckebach, Mol. Phys. 112 (2014) 1773.

[5] M. Iinuma, Y. Takahashi, I. Shake, M. Oda, A. Masaike, T. Yabuzaki, Phys. Rev. Lett. 84 (2000) 171-174.

[6] K. Takeda Triplet State Dynamic Nuclear Polarization (2009) VDM, SaarbrÃijcken, Germany.

[7] A. Kagawa, M. Negoro, K. Takeda, and M. Kitagawa, Rev. Sci. Instr. 80 (2009) 044705.

[8] M. Negoro, K. Tateishi, A. Kagawa, and M. Kitagawa, Phys. Rev. Lett. 107 (2011) 050503.

[9] T. Uesaka, T. Wakui, S. Sakaguchi, Eur. Phys. J. Special Topics 150 (2007) 71-74.

[10] S. Sakaguchi et al., Phys. Rev. C 84 (2011) 024604.

[11] C.P. Brock and J.D. Dunitz, Acta Cryst. B 38 (1982) 2218-2228.

[12] T.R. Eichhorn, M. Haag, B. van den Brandt, P. Hautle, W.Th. Wenckebach, S. Jannin, J.J. Van der Klink, A. Comment, J. Magn. Reson. 234 (2013) 58.

[13] S. Selvakumar, K. Sivaji, A. Arulchakkaravarthi, N. Balamurugan, S. Sankar, P. Ramasam, J. Crystal Growth 282 (2005) 370.

[14] T.R. Eichhorn, N. Niketic, B. van den Brandt, U. Filges, T. Panzner, E. Rantsiou, W.Th. Wenckebach, P. Hautle, Nucl. Instrum. Methods Phys. Res. A 754 (2014) 10.

[15] T.R. Eichhorn, M. Haag, B. van den Brandt, P. Hautle, W.Th. Wenckebach, Chem. Phys. Lett. 555 (2013) 296.

[16] K. Tateishi, M. Negoro, A. Kagawa, and M. Kitagawa, Angew. Chem. Int. Ed. 52 (2013) 13307-13310.

[17] K. Tateishi, M. Negoro, S. Nishida, A. Kagawa, Y. Morita, and M. Kitagawa, PNAS 111 (2014) 7527-7530.

[18] A. Abragam and M. Goldman, Rep. Prog. Phys. 41 (1978) 395.

[19] M. Haag, B. van den Brandt, T.R. Eichhorn, P. Hautle, W.Th. Wenckebach, Nucl. Instrum. Methods Phys. Res. A 678 (2012) 91.

[20] H. Glättli, M. Goldman, in Methods of Experimental Physics (Academic Press, New York, 1987), Vol. 23, part C, pp. 241.

[21] K.L. Krycka, R.A. Booth, C.R. Hogg, Y. Ijiri, J.A. Borchers, et al., Phys. Rev. Lett 104 (2010) 207203.

[22] D. Honecker, A. Ferdinand, F. Döbrich, C.D. Dewhurst, A. Wiedenman, C. Gomez-Polo, K. Suzuki, and A. Michels, Eur. Phys. J. B 76 (2010) 209-213.

[23] A. Michels, D. Honecker, F. Döbrich, C.D. Dewhurst, K. Suzuki, A. Heinemann, Phys. Rev. B 85 (2012) 184417.

[24] N. Niketic, B. van den Brandt, J. Kohlbrecher, W.Th. Wenckebach, P. Hautle, J. Appl. Cryst. 48 (2015) 1514. 